\title{
A 47-Year-Old Man With Rosai-Dorfman Disease
}

\author{
Sara Pereira ${ }^{\mathrm{a}, \mathrm{c}}$, Liliana Oliveira ${ }^{\mathrm{a}}$, Joana Barros ${ }^{\mathrm{a}}$, Hugo Silva ${ }^{\mathrm{b}}$, \\ Carla Melo ${ }^{\text {a }}$, Jorge Salomao ${ }^{a}$, Augusto Duarte ${ }^{\mathrm{a}}$
}

\begin{abstract}
Rosai-Dorfman is a rare and benign disease with unknown etiology, characterized by histiocytosis and emperipolesis, generally associated with lymphadenopathy. It is mainly observed in Caucasians and African children and young adults. A 47-year-old man, Caucasian, with a personal history of chronic alcoholism presented with two lymph nodes with progressive growth over a period of 6 months, one located in the pre-auricular region with $2 \mathrm{~cm}$ and another in the sub-mandibular region with $3 \mathrm{~cm}$. Clinical examination showed two discrete, non-tender, painless and immobile nodes. Biopsy of the nodes was performed. Emperipolesis and cells expressing positive immunoreactivity for protein S-100, CD1a and CD68 were observed making the diagnosis of Rosai-Dorfman disease (RDD). RDD is a benign pathology, self-limited and no special treatment is needed in most cases.
\end{abstract}

Keywords: Rosai-Dorfman disease; Emperipolesis; S-100

\section{Introduction}

Rosai-Dorfman disease (RDD) is a rare pathology observed in 1969 and described in 1972 [1]. It is a benign histiocytic disorder with massive enlargement of lymph nodes, mainly observed in Caucasians and African children and young adults. Although etiology is unknown, it is probably associated to viral infections, like Epstein-Barr, and immunological disorders [2]. The most common clinical presentation of RDD is massive bilateral, cervical, painless, lymphadenopathy sometimes associated with fever, polyclonal hypergam-

\footnotetext{
Manuscript accepted for publication November 22, 2013

${ }^{a}$ Department of Internal Medicine I, Centro Hospitalar Medio Ave, Vila Nova de Famalicao, Portugal

${ }^{\mathrm{b}}$ Department of Family Medicine, USF Nova Era, Porto, Portugal

${ }^{\mathrm{c}}$ Corresponding Author: Sara Pereira, Department of Internal Medicine

I, Centro Hospitalar Medio Ave, Vila Nova de Famalicao, Portugal.

Email: saracp86@gmail.com
}

doi: http://dx.doi.org/10.14740/jmc1599w maglobulinemia and elevated sedimentation rate [2]. However, extranodal disease occurs, even without any clinical involvement of the lymph nodes and the most frequent extranodal sites are skin and soft tissues [3]. The gold standard for diagnosis is biopsy and immunohistochemistry which shows histiocytosis, emperipolesis and immunoreactivity for protein S-100 [4]. RDD is self-limited with good prognosis with spontaneous remission despite the common belief of progressive clinical course [1].

\section{Case Report}

A 47-year-old Caucasian man presented with two cervical nodes with progressive growth over a period of 6 months and was sent to Internal Medicine consultation. He had a personal history of alcohol abuse (more than $100 \mathrm{~g}$ of alcohol per day), chronic gastritis and splenectomy 22 years ago (because of organ trauma). Usual medication included proton pump inhibitor and glucosamine chondroitin. Clinical examination revealed two nodes: one located in the preauricular region with $3 \mathrm{~cm}$ diameter and another in the submandibular region with $2 \mathrm{~cm}$ diameter; non-tender, painless and immobile (Fig. 1). Complete blood test showed hemoglobin $14.6 \mathrm{~g} / \mathrm{dL}$, hematocrit $44.6 \%$, white blood cell count $10,300 / \mathrm{mm}^{3}$, neutrophils $61 \%$, lymphocytes $26.4 \%$, platelets $231,000 / \mathrm{mm}^{3}$ and erythrocyte sedimentation rate of $7 \mathrm{~mm}$ in the first hour. Liver, renal and thyroid function tests were normal and serologic exams for Epstein-Barr virus, cytomegalovirus, $\mathrm{HIV}, \mathrm{HBV}$ and $\mathrm{HVC}$ were negative as well as the diagnostic study for tuberculosis including tuberculin skin test and interferon gamma release assay. Serum protein electrophoresis was normal. Ultrasonography study of the nodes was performed showing adenopathy bulk inside the right parotid gland and further study with biopsy was recommended. That revealed emperipolesis of lymphocytes and cells expressing positive immunoreactivity for protein S-100 highlighted the diagnosis of RDD (Fig. 2). Also, CD68 was positive and $\mathrm{CD} 1 \mathrm{a}$ was negative. $\mathrm{CT}$ of cervical, abdominal and pelvic regions for disease staging was performed. Besides the previously described lesions no other changes were described (Fig. 3). 

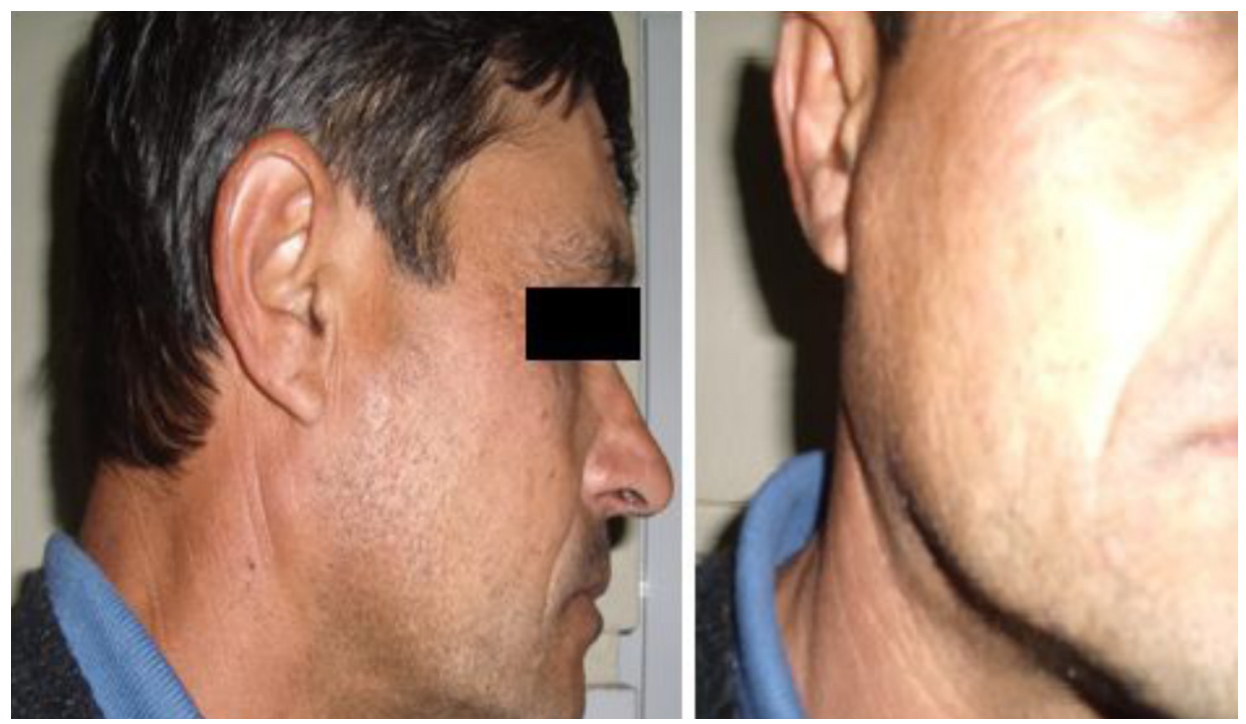

Figure 1. Lateral and frontal view of the parotid nodes.

\section{Discussion}

In 1969, Juan Rosai and Ronald Dorfman reported a histiocytic disorder in several patients with massive enlargement of the lymph nodes [5]. It affects Caucasian children, young adults and African ones. The mean age of presentation is 20.6 years and male to female ratio is 1.4:1 [2]. Etiopatho- genesis of this condition remains unknown but it is believed that it could be associated with viral infections like EpsteinBarr and some immunological disorders related with mononuclear phagocyte and immunoregulatory effector system [1]. The disease was primarily found in lymphatic nodes but extra-lymphatic involvement has also been described.

Typical clinical presentation is cervical lymphadenopa-
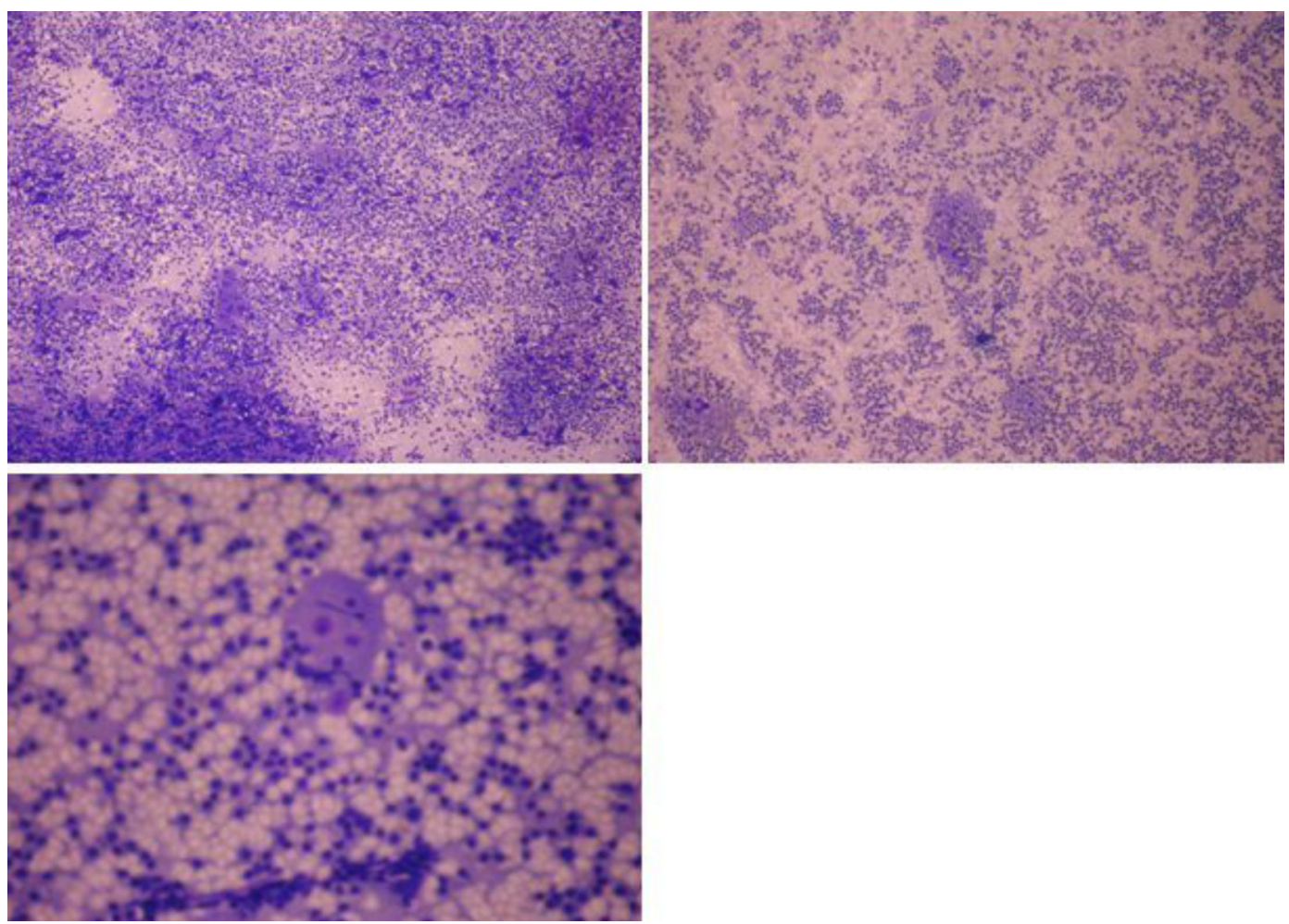

Figure 2. Histological findings showing histiocytosis with emperipolesis. 


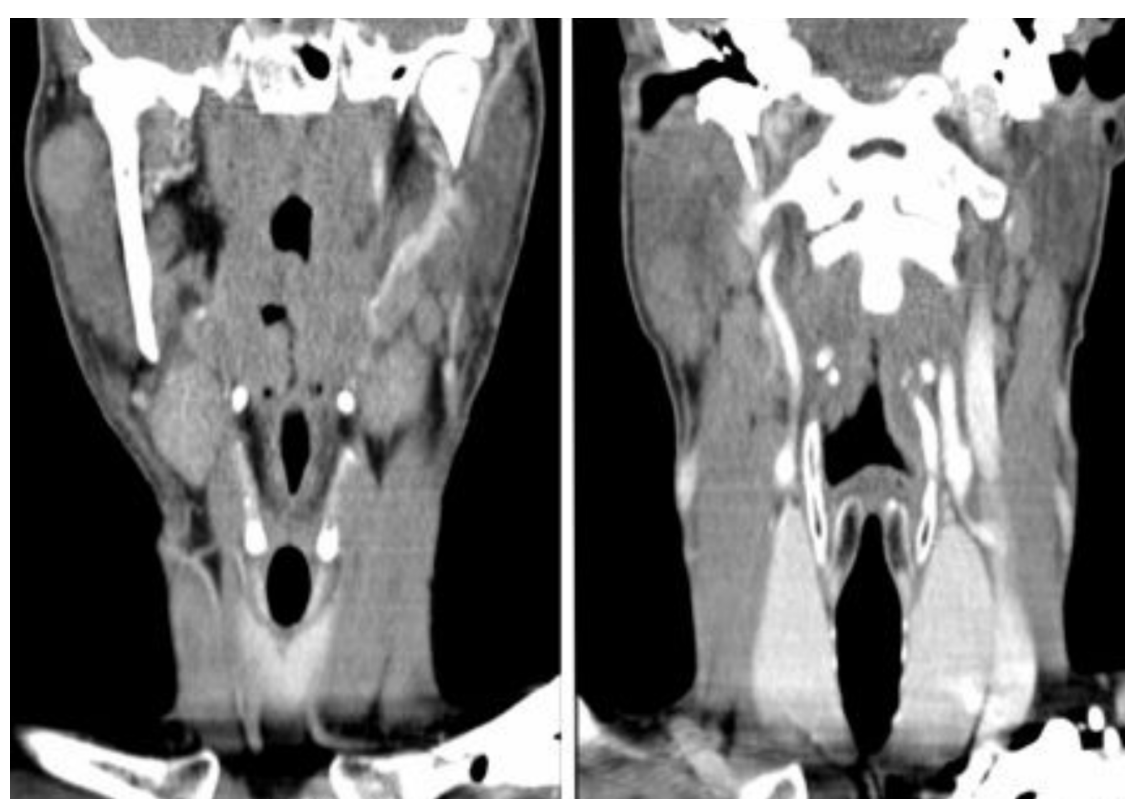

Figure 3. CT cervical scan showing two adenopathy bulks inside the right parotid gland.

thy that represents $87 \%$ of the described cases. The disease has been described also in other locations: outside lymph nodes $(40 \%)$, skin and soft tissue (16\%), nasal cavity (16\%), eyes $(11 \%)$, bones $(11 \%)$, central nervous system $(7 \%)$, salivary glands (7\%), kidney (3\%), airway (respiratory tract) $3 \%$ and liver $1 \%$. In some cases, both lymph nodes and extralymphatic tissues are affected simultaneously [6]. Merely extranodal involvement without significant lymphadenopathy is reported in $23 \%$ of cases [2]. Also, there are some reported cases about RDD that is strictly limited to the skin $[7,8]$.

Clinical findings associated with this disease include fever, sweating at night, malaise and loss of weight [1]. Leukocytosis, neutrophilia, polyclonal hypergammaglobulinemia and elevated erythrocyte sedimentation rate are other associated laboratory findings [2]. In $60 \%$ of patients, hypochromic or normochromic normocytic anemia is reported in published articles.

The gold standard for the diagnosis of RDD is histology of a sample of affected tissue obtained by fine needle aspiration or open biopsy. Dilated sinuses of lymph nodes that are filled with numerous big size histiocytes are observed in the histology. A characteristic finding is emperipolesis of histiocytes in which lymphocytes that are phagocytized by the histiocytes can move in the cytoplasmic vacuoles [9]. Immunochemistry should be performed and reveals positivity for protein S-100. CD68 and alpha-1 antichymotrypsin positivity could also be found [4]. Because of similar clinical and histologic findings, lymphoreticular malignancies such as lymphomas, Hodgkin's disease, malignant histiocytosis and monocytic leukemia should be considered as differential diagnoses [2]. In our case, as Portugal, and particularly northern region, is an endemic region, we also analyze the patient for tuberculosis disease.

Treatment is controversial. Surgery is the most effective treatment for massive lymphadenopathy or extranodal involvement compressing or within vital organs. For the involution of nodes' size, corticosteroids can be used. Other options include chemotherapy, radiotherapy and immunomodulators.

As stated before, prognosis is good, as $70-80 \%$ of the cases showed spontaneous regression of the lesions $[6,10]$. Only in $5-10 \%$ of patients the disease will progress and will rarely have a fatal course [1].

\section{Conclusions}

RDD is a benign pathology, self-limited and no special treatment is needed in most cases. In our case, no treatment was administered and the nodes started to shrink spontaneously.

\section{References}

1. Guven G, Ilgan S, Altun C, Gerek M, Gunhan O. Rosai Dorfman disease of the parotid and submandibular glands: salivary gland scintigraphy and oral findings in two siblings. Dentomaxillofac Radiol. 2007;36(7):428433.

2. Setareh M, Zahra M, Vahid M, Farah S. Generalized lymphadenopathy in infancy; a case report. Iran J Pediatr. 2013;23(1):105-108.

3. Norman L, Bateman AC, Watters GW, Singh V, Spedding AV. Rosai-Dorfman disease presenting as a parotid 
mass. J Laryngol Otol. 1997;111(11):1091-1093.

4. La Barge DV, 3rd, Salzman KL, Harnsberger HR, Ginsberg LE, Hamilton BE, Wiggins RH, 3rd, Hudgins PA. Sinus histiocytosis with massive lymphadenopathy (Rosai-Dorfman disease): imaging manifestations in the head and neck. AJR Am J Roentgenol. 2008;191(6):W299-306.

5. Rosai J, Dorfman RF. Sinus histiocytosis with massive lymphadenopathy. A newly recognized benign clinicopathological entity. Arch Pathol. 1969;87(1):63-70.

6. Histiocytosis Association, a rare community. Available from: www.histio.org.

7. Parrent T, Clark T, Hall D. Cutaneous Rosai-Dorfman disease. Cutis. 2012;90(5):237-238.

8. Wang KH, Chen WY, Liu HN, Huang CC, Lee WR, Hu CH. Cutaneous Rosai-Dorfman disease: clinicopathological profiles, spectrum and evolution of 21 lesions in six patients. Br J Dermatol. 2006;154(2):277-286.

9. Juskevicius R, Finley JL. Rosai-Dorfman disease of the parotid gland: cytologic and histopathologic findings with immunohistochemical correlation. Arch Pathol Lab Med. 2001;125(10):1348-1350.

10. Vuong V, Moulonguet I, Cordoliani F, Crickx B, Bezier M, Vignon-Pennamen MD, Flageul B, et al. [Cutaneous revelation of Rosai-Dorfman disease: 7 cases]. Ann Dermatol Venereol. 2013;140(2):83-90. 